\title{
A New Method for Generating Discrete Analogues of Continuous Distributions
}

\author{
M. Ganji and F. Gharari \\ Department of statistics, University of Mohaghegh Ardabili, Ardabil, Iran. \\ E-mails: mganji@uma.ac.ir, f.gharari@uma.ac.ir
}

\begin{abstract}
In this paper we use discrete fractional calculus for showing the existence of delta and nabla discrete distributions and then apply time scales for definition of delta and nabla discrete gamma distributions. The main result of this paper is unification of the continuous and discrete gamma distributions, which is at the same time a distribution to so-called time scale. Also, starting from the Laplace transform on time scales, we develop concept of moment generating function for these distributions.
\end{abstract}

Keywords: Discrete fractional calulus, Time scale, Gamma distribution Subjclass: 60E05, 93C70, 26A33, 39A12.

Received 9 May 2017

\section{Introduction}

Accepted 12 September 2017

One of the active areas of research in statistics is to model discrete life time data by developing discretized version of suitable continuous lifetime distributions. The discretization of a continuous distribution using different methods has attracted renewed attention of researchers in last few years, for example, see $[3,8,12,13,14,15,16,17]$. Recently, these different methods are classified based on different criteria of discretization in detail by Chakraborty [9].

In this article, we present a new method for discretization of most of continuous distributions, where their probability density functions (pdfs) consist of the monomial Taylor and exponential function, and as an example we do discretization for gamma distribution with this method. Our discretization method, in comparison with prior methods for discretization of continuous distributions, has two main advantages. First, for a given continuous distribution, it is possible to generate two types (delta and nabla types) of corresponding discrete distributions. Second, the unification of the continuous distribution and corresponding discrete distributions, which is at the same time a distribution to the case of a time scale. We use discrete fractional calculus for showing the existence of delta and nabla discrete distributions and then apply time scales for definition of delta and nabla discrete distributions and as an unification theory under which continuous and discrete distributions are subsumed. 
The article is organized as follows: The second section contains summary of some notations and definitions in delta and nabla calculus, also the definitions of delta and nabla Riemann right fractional sums and differences. In the third section, we use discrete fractional calculus for showing the existence of delta and nabla discrete distributions. In the fourth and fifth sections, we define novel types of moments for delta and nabla discrete distributions and provide a method for obtaining these moments using the Laplace transform on the discrete time scale. The sixth section contains an unification of ordinary moments and delta and nabla moments, also types of the mgfs. In section 7 , delta and nabla discrete gamma distributions is defined. While section 8 contains an unification of discrete and continuous gamma distributions. In the final section an application of the proposed distribution is presented.

\section{$2 \quad$ Preliminaries}

In this section, we provide a collection of definitions and related results which are essential and will be used in the next discussions. As mentioned in $[5,6]$, the definitions and theorem are as following.

A time scale $\mathbb{T}$ is an arbitrary nonempty closed subset of the real numbers $\mathbb{R}$. The most well-known examples are $\mathbb{T}=\mathbb{R}$ and $\mathbb{T}=\mathbb{Z}$. The forward (backward) jump operator is defined by $\sigma(t):=\inf \{s \in \mathbb{T}: s>t\}(\rho(t):=\sup \{s \in \mathbb{T}: s<t\})$, where inf $\emptyset:=\sup \mathbb{T}$ and $\sup \emptyset:=i n f \mathbb{T}$. A point $t \in \mathbb{T}$ is said to be right-dense if $t<\sup \mathbb{T}$ and $\sigma(t)=t$ (leftdense if $t>$ inf $\mathbb{T}$ and $\rho(t)=t$ ), right-scattered if $\sigma(t)>t \quad($ left-scattered if $\rho(t)<t)$. The forward (backward) graininess function $\mu: \mathbb{T} \rightarrow[0, \infty)(\nu: \mathbb{T} \rightarrow[0, \infty))$ is defined by $\mu(t):=\sigma(t)-t(\nu(t):=t-\rho(t))$. More generally, we will denote all $\rho(t), \sigma(t)$ and $t$ with $\eta(t)$.

Definition 2.1. A function $f: \mathbb{T} \rightarrow \mathbb{R}$ is called regulated if its right-sided limits exist at all right-dense points in $\mathbb{T}$ and its left-sided lim its exist at all left-dense points in $\mathbb{T}$.

Definition 2.2. A function $f: \mathbb{T} \rightarrow \mathbb{R}$ is called rd-continuous (ld-continuous) if it is continuous at right-dense (left-dense) points in $\mathbb{T}$ and its left-sided (right-sided) limits exist at left-dense (right-dense) points in $\mathbb{T}$.

The set $\mathbb{T}_{k}\left(\mathbb{T}_{k}^{*}\right)$ is derived from the time scale $\mathbb{T}$ as follows: If $\mathbb{T}$ has a left-scattered maximum (right-scattered minimum) $m$, then $\mathbb{T}_{k}:=\mathbb{T}-\{m\} \quad\left(\mathbb{T}_{k}^{*}:=\mathbb{T}-\{m\}\right)$. Otherwise, $\mathbb{T}_{k}:=\mathbb{T}\left(\mathbb{T}_{k}^{*}:=\mathbb{T}\right)$.

Definition 2.3. A function $f: \mathbb{T} \rightarrow \mathbb{R}$ is said to be delta (nabla) differentiable at a point $t \in \mathbb{T}_{k}\left(t \in \mathbb{T}_{k}^{*}\right)$ if there exists a number $f^{\Delta}(t)\left(f^{\nabla}(t)\right)$ with the property that given any $\epsilon>0$, there exists a neighborhood $U$ of $t$ such that

$$
\begin{array}{r}
\left|f(\sigma(t))-f(s)-f^{\Delta}(t)(\sigma(t)-s)\right| \leq \epsilon|\sigma(t)-s| \\
\left(\left|f(\rho(t))-f(s)-f^{\nabla}(t)(\rho(t)-s)\right| \leq \epsilon|\rho(t)-s|\right)
\end{array}
$$

for all $s \in U$. 
For a function $f: \mathbb{T} \rightarrow \mathbb{R}$ it is possible to introduce a derivative $f^{\Delta}(t)\left(f^{\nabla}(t)\right)$ and an integral $\int_{a}^{b} f(t) \Delta t\left(\int_{a}^{b} f(t) \nabla t\right)$ in such a manner that $f^{\Delta}(t)=f^{\prime}\left(f^{\nabla}(t)=f^{\prime}\right)$ and $\int_{a}^{b} f(t) \Delta t=\int_{a}^{b} f(t) d t\left(\int_{a}^{b} f(t) \nabla t=\int_{a}^{b} f(t) d t\right)$ in the case $\mathbb{T}=\mathbb{R}$ and $f^{\Delta}(t)=$ $\Delta f\left(f^{\nabla}(t)=\nabla f\right)$ and $\int_{a}^{b} f(t) \Delta t=\sum_{a}^{b-1} f(t)\left(\int_{a}^{b} f(t) \nabla t=\sum_{a+1}^{b} f(t)\right)$ in the case $\mathbb{T}=\mathbb{Z}$, where, the forward and backward difference operators are defined by $\Delta f=$ $f(t+1)-f(t)$ and $=f(t)-f(t-1)$, respectively. Also, we define the iterated operators $\Delta^{n}=\Delta\left(\Delta^{n-1}\right)$ and $\nabla^{n}=\nabla\left(\nabla^{n-1}\right)$ for $n \in \mathbb{N}$.

Definition 2.4. A function $p: \mathbb{T} \rightarrow \mathbb{R}$ is called $\mu$-regressive ( $\nu$-regressive) provided $1+\mu(t) p(t) \neq o(1-\nu(t) p(t) \neq o)$ for all $t \in \mathbb{T}_{k}\left(t \in \mathbb{T}_{k}^{*}\right)$.

The set $\mathbb{R}_{\mu}\left(\mathbb{R}_{\nu}\right)$ of all $\mu$-regressive and rd-continuous ( $\nu$-regressive and ld-continuous) functions forms an Abelian group under the circle plus addition $\bigoplus$ defined by $(p \bigoplus q)(t):=$ $p(t)+q(t)+\mu(t) p(t) q(t) \quad((p \bigoplus q)(t):=p(t)+q(t)-\nu(t) p(t) q(t))$ for all $t \in \mathbb{T}_{k}\left(t \in \mathbb{T}_{k}^{*}\right)$. The additive inverse $\ominus p$ of $p \in \mathbb{R}_{\mu}\left(p \in \mathbb{R}_{\nu}\right)$ is defined by

$$
(\ominus p)(t):=-\frac{p(t)}{1+\mu(t) p(t)} \quad\left((\ominus p)(t):=-\frac{p(t)}{1-\nu(t) p(t)}\right)
$$

for all $t \in \mathbb{T}_{k}\left(t \in \mathbb{T}_{k}^{*}\right)$.

For real numbers $a$ and $b$ we denote $\mathbb{N}_{a}=\{a, a+1, \ldots\}$ and ${ }_{b} \mathbb{N}=\{b, b-1, \ldots\}$.

Theorem 2.5. Let $p \in \mathbb{R}_{\mu}\left(p \in \mathbb{R}_{\nu}\right)$ and $t_{0} \in \mathbb{T}$ be a fixed point. Then the delta (nabla) exponential function $e_{p}\left(., t_{0}\right)\left(e_{p}^{*}\left(., t_{0}\right)\right)$ is the unique solution of the initial value problem

$$
y^{\Delta}=p(t) y, \quad y\left(t_{0}\right)=1 \quad\left(y^{\nabla}=p(t) y, \quad y\left(t_{0}\right)=1\right) .
$$

If $\mathbb{T}=\mathbb{N}_{a}$, when $p(t) \equiv p$, where $p \in \mathbb{R}_{\mu}\left(p \in \mathbb{R}_{\nu}=\mathbb{C} \backslash\{1\}\right)$ and $t_{0}=a$, it is easy to see that $e_{p}(t, a)=(1+p)^{t-a}\left(e_{p}^{*}(t, a)=(1-p)^{a-t}\right)$ and if $\mathbb{T}=\mathbb{R}, e_{p}(t, a)=e^{p(t-a)}\left(e_{p}^{*}(t, a)=\right.$ $\left.e^{p(t-a)}\right)$, where " $e^{"}$ is ordinary exponential function. Moreover, in the special case, $e_{1}(t, 0)=2^{t}\left(e_{\frac{1}{2}}^{*}(t, 0)=2^{t}\right)$. More generally, we will denote all $e_{p}(t, a), e_{p}^{*}(t, a)$ and $e^{p(t-a)}$ with $\hat{e}_{p}(t, a)$.

Definition 2.6. The delta (nabla) Taylor monomials are the functions $h_{n}: \mathbb{T} \times \mathbb{T} \rightarrow$ $\mathbb{R}, n \in \mathbb{N}_{0}$, and are defined recursively as follows:

$$
\begin{array}{lll}
h_{0}(t, s)=1, & h_{n+1}(t, s)=\int_{s}^{t} h_{n}(\tau, s) \Delta \tau & \forall t, s \in \mathbb{T} \\
\left(h_{0}^{*}(t, s)=1,\right. & h_{n+1}^{*}(t, s)=\int_{s}^{t} h_{n}^{*}(\tau, s) \nabla \tau & \forall t, s \in \mathbb{T}) .
\end{array}
$$

We consider three cases for the time scale $\mathbb{T}$.

(a) If $\mathbb{T}=\mathbb{R}$, then $\sigma(t)=\rho(t)=t$ and the Taylor monomials can be written explicitly as

$$
h_{n}(t, s)=h_{n}^{*}(t, s)=\frac{(t-s)^{n}}{n !}, \quad t, s \in \mathbb{R}, n \in \mathbb{N}_{0} .
$$

For each $\alpha \in \mathbb{R} \backslash\{-\mathbb{N}\}$ define the $\alpha$-th Taylor monomial to be

$$
h_{\alpha}(t, s)=\frac{(t-s)^{\alpha}}{\Gamma(\alpha+1)}
$$


and $\Gamma$ denoted the special gamma function.

In this paper, we only consider the special case, $h_{\alpha}(t):=h_{\alpha}(t, 0)=\frac{t^{\alpha}}{\Gamma(\alpha+1)}$ as Taylor monomial $(\mathrm{tm})$.

(b) If $\mathbb{T}=\mathbb{Z}$, then $\sigma(t)=t+1$ and the Taylor monomials can be written explicitly as

$$
h_{n}(t, s)=\frac{(t-s)^{\underline{n}}}{n !}, \quad t, s \in \mathbb{Z}, n \in \mathbb{N}_{0},
$$

where $t^{\underline{n}}=\Pi_{j=0}^{n-1}(t-j)=\frac{\Gamma(t+1)}{\Gamma(t+1-j)}$ and product is zero when $t+1-j=0$ for some $j$. More generally, for $\alpha$ arbitrary define $t^{\underline{\alpha}}=\frac{\Gamma(t+1)}{\Gamma(t+1-\alpha)}$, where the convention of that division at pole yields zero. This generalized falling function allows us to extend (2.5) to define a general Taylor monomial that will serve us well in the probability distributions setting.

For each $\alpha \in \mathbb{R} \backslash\{-\mathbb{N}\}$, define the delta $\alpha$-th Taylor monomial to be

$$
h_{\alpha}(t, s)=\frac{(t-s)^{\underline{\alpha}}}{\Gamma(\alpha+1)} \text {. }
$$

In this paper, we only consider the special case $h_{\underline{\alpha}}(t):=h_{\alpha}(t, 0)=\frac{t^{\underline{\alpha}}}{\Gamma(\alpha+1)}$ as delta Taylor monomial $(d t m)$.

(c) If $\mathbb{T}=\mathbb{Z}$, then $\rho(t)=t-1$ and the Taylor monomials can be written explicitly as

$$
h_{n}(t, s)=\frac{(t-s)^{\bar{n}}}{n !}, \quad t, s \in \mathbb{Z}, n \in \mathbb{N}_{0},
$$

where $t^{\bar{n}}=\prod_{j=0}^{n-1}(t+j)=\frac{\Gamma(t+n)}{\Gamma(t)}$. More generally, for any real number $\alpha$ rising function is defined as $t^{\bar{\alpha}}=\frac{\Gamma(t+\alpha)}{\Gamma(t)}$ where $t \in \mathbb{R} \backslash\left\{-\mathbb{N}_{0}\right\}$ and $0^{\bar{\alpha}}=0$. This function allows us to extend (2.7) in order to define a general Taylor monomial that will serve us well in the probability distributions setting.

For each $\alpha \in \mathbb{R} \backslash\{-\mathbb{N}\}$ define the nabla $\alpha$-th Taylor monomial to be

$$
h_{\alpha}^{*}(t, s)=\frac{(t-s)^{\bar{\alpha}}}{\Gamma(\alpha+1)} .
$$

In this paper, we only consider the special case $h_{\bar{\alpha}}(t):=h_{\alpha}^{*}(t, 0)=\frac{t^{\bar{\alpha}}}{\Gamma(\alpha+1)}$ as nabla Taylor monomial $(\mathrm{ntm})$.

More generally, we will denote all $h_{\underline{\alpha}}(t), h_{\bar{\alpha}}(t)$ and $h_{\alpha}(t)$ with $\hat{h}_{\alpha}(t)$.

Definition 2.7. The delta (nabla) Laplace transform of a regulated function $f: \mathbb{T}_{a} \rightarrow \mathbb{R}$ is given by

$$
\mathbf{L}_{a}\{f\}(s)=\int_{a}^{\infty} e_{\ominus s}(\sigma(t), a) f(t) \Delta t \quad\left(\mathbf{L}_{a}^{*}\{f\}(s)=\int_{a}^{\infty} e_{\ominus s}^{*}(\rho(t), a) f(t) \nabla t\right),
$$

for all $s \in \mathcal{D}\{f\}$, where $a \in \mathbb{R}$ is fixed, $\mathbb{T}_{a}$ is an unbounded time scale with infimum $a$ and $\mathcal{D}\{f\}$ is the set of all regressive complex constants for which the integral converges. 
In the special case, when $\mathbb{T}=\mathbb{N}$, every function is regulated and its delta (nabla) discrete Laplace transform can be written as

$$
\mathbf{L}_{a}\{f\}(s)=\sum_{t=a}^{\infty}\left(\frac{1}{1+s}\right)^{\sigma(t)} f(t) \quad\left(\mathbf{L}_{a}^{*}\{f\}(s)=\sum_{t=a}^{\infty}(1-s)^{\rho(t)} f(t)\right) .
$$

Let $b$ be a real number and $f:{ }_{b} \mathbb{N} \longrightarrow \mathbb{R}$. The delta Riemann left fractional sum of order $\alpha>0$ is defined by Abdeljawad [1] as

$$
\Delta^{-\alpha} f(t)=\frac{1}{\Gamma(\alpha)} \sum_{s=t+\alpha}^{b}(\rho(s)-t)^{\frac{\alpha-1}{}} f(s), \quad t \in{ }_{b-\alpha} \mathbb{N} .
$$

We define the nabla Riemann right fractional sum of order $\alpha>0$ as

$$
\nabla^{-\alpha} f(t)=\frac{1}{\Gamma(\alpha)} \sum_{s=t}^{b-1}(\sigma(s)-t)^{\overline{\alpha-1}} f(s), \quad t \in{ }_{b-1} \mathbb{N} .
$$

The delta Riemann right fractional difference of order $\alpha>0$ is defined by Abdeljawad [1] as

$$
\Delta^{\alpha} f(t)=(-1)^{n} \nabla^{n} \Delta^{-(n-\alpha)} f(t)
$$

for $t \in{ }_{b-(n-\alpha)} \mathbb{N}$ and $n=[\alpha]+1$ where $[\alpha]$ is the greatest integer less than $\alpha$. Also, the nabla Riemann right fractional difference of order $\alpha>0$ is defined by

$$
\nabla^{\alpha} f(t)=(-1)^{n} \Delta^{n} \nabla^{-(n-\alpha)} f(t)
$$

for $t \in{ }_{b-n} \mathbb{N}$.

In [2], author obtained the following alternative definition for delta Riemann right fractional difference

$$
\Delta^{\alpha} f(t)=\frac{1}{\Gamma(-\alpha)} \sum_{s=t-\alpha}^{b}(\rho(s)-t)^{\frac{-\alpha-1}{2}} f(s) .
$$

Similarly, we can prove the following formula for nabla Riemann right fractional difference

$$
\nabla^{\alpha} f(t)=\frac{1}{\Gamma(-\alpha)} \sum_{s=t}^{b-1}(\sigma(s)-t)^{\overline{-\alpha-1}} f(s) .
$$

For an introduction to discrete fractional calculus the reader is referred to [11].

\section{Generating discrete distributions by discrete frac- tional calculus}

The following results show the relationship between continuous and discrete fractional calculus and statistics and also allows us to define different types of discrete distributions. 
Suppose that $X$ is a positive continuous random variable. The expectation of the $\mathrm{tm}$ function, $h_{\alpha-1}(X)$, coincides with Riemann-Liouville right fractional integral of the pdf at the origin for $\alpha>0$ and Marchaud right fractional derivative of the pdf at the origin for $-1<\alpha<0$, that is, we have

$$
E\left[h_{\alpha-1}(X)\right]=\left\{\begin{array}{lr}
\left(I_{-}^{\alpha} f\right)(0), & \alpha>0 \\
\left(D_{-}^{\alpha} f\right)(0), & -1<\alpha<0
\end{array}\right.
$$

where

$$
\left(I_{-}^{\alpha} f\right)(t)=\frac{1}{\Gamma(\alpha)} \int_{0}^{\infty} x^{\alpha-1} f(x+t) d x
$$

is the Riemann-Liouville right fractional integral, while

$$
\left(D_{-}^{\alpha} f\right)(t)=\frac{1}{\Gamma(-\alpha)} \int_{0}^{\infty} x^{-\alpha-1}\{f(x+t)-f(t)\} d x
$$

is the Marchaud left fractional derivative[10].

It can be seen that the limits of the above integrals equal to the support of random variable $X$. Considering this point, we present the following theorems for discrete random variable $X$.

Theorem 3.1. Suppose that $X$ is a discrete random variable. The expectation of the dtm function, $h_{\underline{\alpha-1}}(X)$, coincides with delta Riemann right fractional sum of the pmf at -1 for $\alpha>0$ and delta Riemann right fractional difference of the probability mass function (pmf) at -1 for $\alpha<0, \alpha \notin\{-\mathbb{N}\}$, i.e.

$$
E\left[h_{\underline{\alpha-1}}(X)\right]=\left\{\begin{array}{lr}
\left(\Delta^{-\alpha} f\right)(-1), & \alpha>0 \\
\left(\Delta^{\alpha} f\right)(-1), & \alpha<0, \alpha \notin\{\ldots,-2,-1\}
\end{array}\right.
$$

where

$$
\left(\Delta^{-\alpha} f\right)(t)=\sum_{x=\alpha-1}^{b-1-t} \frac{x \frac{\alpha-1}{\Gamma(\alpha)}}{\Gamma(x+t+1)}
$$

is the delta Riemann right fractional sum, while

$$
\left(\Delta^{\alpha} f\right)(t)=\sum_{x=-\alpha-1}^{b-t-1} \frac{x-\alpha-1}{\Gamma(-\alpha)} f(x+t+1)
$$

is the delta Riemann right fractional difference.

Proof. For $\alpha>0$, substitute $x=\rho(s)-t$ in the expression (2.10) and also for $\alpha<0$ and $\alpha \notin\{-1,-2, \ldots\}$, in the expression (2.12).

Here, considering the limits of summation we can define the discrete distributions with the support $\mathbb{N}_{\alpha-1}$ or a finite subset of it. In this case, we will call $X$, delta discrete random variable. As an example, we will define the delta discrete gamma distribution. Another example is the delta discrete uniform distribution, $D U\{\alpha-1, \alpha, \ldots, \alpha+\beta\}$, where $\alpha \in \mathbb{R}$ and $\beta \in \mathbb{N}_{-1}$. 
Theorem 3.2. We suppose that $X$ is a discrete random variable. The expectation of the ntm function, $h_{\overline{\alpha-1}}(X)$, coincides with nabla Riemann right fractional sum of the pmf at 1 for $\alpha>0$ and nabla Riemann right fractional difference of the pmf at 1 for $\alpha<0, \alpha \notin\{-\mathbb{N}\}$, i.e.

$$
E\left[h_{\overline{\alpha-1}}(X)\right]=\left\{\begin{array}{lr}
\left(\nabla^{-\alpha} f\right)(1), & \alpha>0 \\
\left(\nabla^{\alpha} f\right)(1), & \alpha<0, \alpha \notin\{\ldots,-2,-1\}
\end{array}\right.
$$

where

$$
\left(\nabla^{-\alpha} f\right)(t)=\sum_{x=1}^{b+1-t} \frac{x^{\overline{\alpha-1}}}{\Gamma(\alpha)} f(x+t-1)
$$

is the nabla Riemann right fractional sum, while

$$
\left(\nabla^{\alpha} f\right)(t)=\sum_{x=1}^{b+1-t} \frac{x^{\overline{-\alpha-1}}}{\Gamma(-\alpha)} f(x+t-1)
$$

is the nabla Riemann right fractional difference.

Proof. For $\alpha>0$, substitute $x=\sigma(s)-t$ in the expression (2.11) and also for $\alpha<0$ and $\alpha \notin\{-1,-2, \ldots\}$, in the expression $(2.13)$.

Therefore, considering the limits of summation in recent theorem, we can define the discrete distributions with support $\mathbb{N}_{1}$ or a finite subset of it. In this case, we will call $X$, nabla discrete random variable. In this work, we will define the nabla discrete gamma distribution. Another example is the nabla discrete uniform distribution, $D U\{1,2, \ldots, \alpha-\beta+1\}$, where $\alpha \in \mathbb{R}$ and $\beta \in{ }_{\alpha} \mathbb{N}$.

\section{Nabla moments and nabla moment generating function}

In this section, we define novel types of moments for delta and nabla discrete distributions and provide a method for obtaining these moments using the Laplace transform on the discrete time scale.

It is well known that the Laplace transform of pdf is the moment generating function (mgf), which is defined as $M_{X}(-t)=E\left[e^{-t X}\right]=\int_{0}^{\infty} e^{-t x} f(x) d x$, and $X$ is a non- negative real-valued random variable and $t$ is a complex variable with non-negative real part. On the other hand, it can be easily seen that $M_{X}(-t)=\sum_{k=0}^{\infty} E\left[(-X)^{k}\right] \frac{t^{k}}{k !}$. This function generates the moments of integer order of random variable $X$ as $\mu_{k}=E\left[X^{k}\right]=$ $\left.(-1)^{k} \frac{d^{k} M_{X}(-t)}{d t^{k}}\right|_{t=0}$.

Now, suppose that $X$ is a delta discrete random variable with values $x=\mathbb{N}_{\alpha-1}, \alpha>0$.

The delta discrete Laplace transform of pmf of $X$ is defined as

$$
\sum_{x=\alpha-1}^{\infty}\left(\frac{1}{1+t}\right)^{\sigma(x)} f(x)=E\left[(1+t)^{-\sigma(x)}\right]=E\left[e_{\ominus t}(\sigma(X), 0)\right]=M_{\sigma(X)}(-t),
$$


and $t$ is the set of all regressive complex constants for which the series converges. By using the series expansion for $(1+t)^{-x}$ it can be easily proved that $M_{\sigma(X)}(-t)=$ $\sum_{k=0}^{\infty} E\left[(-1)^{k}(\sigma(X))^{\bar{k}}\right] \frac{t^{k}}{k !}$. This function generates the nabla moments of integer order of $X$ as $E\left[(\sigma(X))^{K}\right]=\left.(-1)^{k} \frac{d^{k} M_{\sigma(X)}(-t)}{d t^{k}}\right|_{t=0}$.

Definition 4.1. Let $X$ be a delta discrete random variable with pmf $f$.

(a) Its $k$-th nabla moment, is denoted by $\mu_{k}^{\nabla}$ and is defined by $\mu_{k}^{\nabla}:=\sum_{x}(\sigma(X))^{\bar{k}} f(x)$.

(b) The nabla mgf of $X$ is given by $M_{\sigma(X)}(t)=E\left[e_{t}^{*}(\sigma(X), 0)\right]$.

Theorem 4.2. Let $X$ be a delta discrete random variable with nabla moments $\mu_{k}^{\nabla}$. we have

$$
M_{\sigma(X)}(t)=\sum_{k=0}^{\infty} E\left[(\sigma(X))^{K}\right] \frac{t^{k}}{k !} .
$$

In particular,

$$
E\left[(\sigma(X))^{\bar{K}}\right]=\left.\frac{d^{k} M_{\sigma(X)}(t)}{d t^{k}}\right|_{t=0}
$$

Proof. For the proof of (4.1), we use the series expansion for function $(1-t)^{-x}$, that is $\sum_{k=0}^{\infty} x^{\underline{k}} \frac{t^{k}}{k !}$. we have

$$
M_{\sigma(X)}(t)=\sum_{x} \sum_{k=0}^{\infty}(\sigma(X))^{\bar{k}} \frac{t^{k}}{k !} f(x)=\sum_{k=0}^{\infty}\left(\sum_{x}(\sigma(X))^{\bar{k}} f(x)\right) \frac{t^{k}}{k !} .
$$

For the proof (4.2), differentiate $M_{\sigma(X)}(t)$ a total of $k$ times. Since the only $t$ - dependence in the summation is the $(1-t)^{-\sigma(x)}$ factor, we have

$$
\frac{d^{k}}{d t^{k}} M_{\sigma(X)}(t)=\sum_{x}\left[\frac{d^{k}}{d t^{k}}(1-t)^{-\sigma(x)}\right] f(x)=\sum_{x}(\sigma(X))^{\bar{k}}(1-t)^{-(\sigma(x)+k)} f(x),
$$

the claim now follows from taking $t=0$ and recalling the definition of the nabla moments.

\section{Delta moments and delta moment generating func- tion}

Suppose that $X$ is a nabla discrete random variable with values $x=\mathbb{N}_{1}$. The nabla discrete Laplace transform of pmf of $X$ is defined as

$$
\sum_{x=1}^{\infty}(1-t)^{\rho(x)} f(x)=E\left[(1-t)^{\rho(x)}\right]=E\left[e_{\ominus t}^{*}(\rho(X), 0)\right]=M_{\rho(X)}(-t)
$$

and $t$ is the set of all regressive complex constants for which the series converges. By using the series expansion for $(1-t)^{x}$, it can be easily proved that $M_{\rho(X)}(-t)=$ $\sum_{k=0}^{\infty} E\left[(-1)^{k}(\rho(X))^{\underline{k}}\right] \frac{t^{k}}{k !}$. This function generates the delta moments of integer order of $X$ as $E\left[(\rho(X))^{\underline{K}}\right]=\left.(-1)^{k} \frac{d^{k} M_{\rho(X)}(-t)}{d t^{k}}\right|_{t=0}$. 
Definition 5.1. Let $X$ be a nabla discrete random variable with $\operatorname{pmf} f$.

(a) Its $k$-th delta moment, denoted by $\mu_{k}^{\Delta}$ and is defined by $\mu_{k}^{\Delta}:=\sum_{x}(\rho(X))^{\underline{k}} f(x)$.

(b) The delta mgf of $X$ is given by $M_{\rho(X)}(t)=E\left[e_{t}(\rho(X), 0)\right]$.

Theorem 5.2. Let $X$ be a nabla discrete random variable with delta moments $\mu_{k}^{\Delta}$. we have

$$
M_{\rho(X)}(t)=\sum_{k=0}^{\infty} E\left[(\rho(X))^{\underline{\underline{K}}}\right] \frac{t^{k}}{k !}
$$

In particular,

$$
E\left[(\rho(X))^{\underline{K}}\right]=\left.\frac{d^{k} M_{\rho(X)}(t)}{d t^{k}}\right|_{t=0}
$$

Proof. The proof is similar to the proof of theorem 4.2. We only outline this point that $(1+t)^{x}=\sum_{k=0}^{\infty} x^{\underline{k}} \frac{t^{k}}{k !}$.

More generally, we denote all the $\mu_{k}^{\Delta}, \mu_{k}^{\nabla}$ and $\mu_{k}$ with $\hat{\mu}_{k}$ and also all the $M_{\sigma(X)}(t), M_{\rho(X)}(t)$ and $M_{X}(t)$ with $M_{\eta(X)}(t)$, which are useful notations in statistics.

\section{$6 \quad$ Unification of the mgf and moments}

For a given time scale $\mathbb{T}$, we present the construction of moments and the mgfs on time scales as

$$
\hat{\mu}_{k}=E\left[\Gamma(k+1) \hat{h}_{k}(\eta(X))\right] \text { and } M_{\eta(X)}(-t)=E\left[\hat{e}_{\ominus t}(\eta(X), 0)\right], \quad x \in \mathbb{T}
$$

,respectively. In order that, the reader sees how ordinary moments and delta and nabla moments, also types of the mgfs follow from (6.1), it is only at this point necessary to know that

$$
\hat{h}_{k}(x)=h_{k}(x)=\frac{x^{k}}{\Gamma(k+1)}, \eta(x)=\sigma(x)=\rho(x)=x \text { and } \hat{e}_{\ominus t}(\eta(X), 0)=e^{-t x},
$$

if $\mathbb{T}=\mathbb{R}^{+}$,

$$
\hat{h}_{k}(x)=h_{\underline{k}}(x)=\frac{x^{\underline{k}}}{\Gamma(k+1)}, \quad \eta(x)=\sigma(x) \quad \text { and } \quad \hat{e}_{\ominus t}(\eta(X), 0)=(1+t)^{-\sigma(x)},
$$

if $\mathbb{T}=\mathbb{N}_{\alpha-1}, \alpha>0$ and

$$
\hat{h}_{k}(x)=h_{\bar{k}}(x)=\frac{x^{\bar{k}}}{\Gamma(k+1)}, \quad \eta(x)=\rho(x) \quad \text { and } \quad \hat{e}_{\ominus t}(\eta(X), 0)=(1-t)^{\rho(x)}
$$

if $\mathbb{T}=\mathbb{N}_{1}$. 


\section{$7 \quad$ The delta and nabla discrete gamma distributions}

In this section, we will introduce delta and nabla discrete gamma distributions, by substituting continuous Taylor monomials and exponential functions with their corresponding discrete types (on the discrete time scale) in continuous gamma distribution.

\subsection{The delta discrete gamma distribution}

Definition 7.1. It is said that the random variable $X$ has a delta discrete gamma distribution with $(\alpha, \beta)$ parameters if its pmf is given by

$$
\operatorname{Pr}[X=x]=\frac{h_{\underline{\alpha-1}}(x) \beta^{\alpha}}{e_{\beta}(\sigma(x), 0)}=\frac{x^{\underline{\alpha-1}} \beta^{\alpha}}{\Gamma(\alpha)(1+\beta)^{\sigma(x)}}, \quad x=\mathbb{N}_{\alpha-1},
$$

where $\alpha>0, \beta>0$ and it denotes as $\Gamma^{\Delta}(\alpha, \beta)$.

- Particular cases:

(a) For $\alpha=1, \Gamma^{\Delta}(\alpha, \beta)$ in (7.1) reduces to a one parameter delta discrete gamma or delta exponential distribution, $\Gamma^{\Delta}(1, \beta) \equiv E^{\Delta}(\beta)$ with pmf

$$
\operatorname{Pr}[X=x]=\beta(1+\beta)^{-\sigma(x)}=\left(\frac{\beta}{1+\beta}\right)\left(\frac{1}{1+\beta}\right)^{x}, \quad x=0,1, \ldots
$$

Obviously, this is the pmf of geometric distribution (the number of failures for first success).

(b) For $\alpha=n, n \in \mathbb{N}, \Gamma^{\Delta}(\alpha, \beta)$ in (7.1) is a delta discrete Erlang distribution $\Gamma^{\Delta}(n, \beta)$ with pmf

$$
\operatorname{Pr}[X=x]=\left(\begin{array}{c}
x-n+1 \\
x
\end{array}\right) \beta^{n}(1+\beta)^{-\sigma(x)}, \quad x=\mathbb{N}_{n-1} .
$$

If we substitute $\sigma(x)=x,(7.2)$ and (7.3) are given by

$$
\operatorname{Pr}[X=x]=\left(\frac{\beta}{1+\beta}\right)\left(\frac{1}{1+\beta}\right)^{x-1}, \quad x=1,2, \ldots,
$$

and

$$
\operatorname{Pr}[X=x]=\left(\begin{array}{c}
x-n \\
x-1
\end{array}\right)\left(\frac{\beta}{1+\beta}\right)^{n}\left(\frac{1}{1+\beta}\right)^{x-n}, \quad x=n, n+1, \ldots
$$

respectively. It can be seen that (7.5) is the same negative binomial distribution (the number of independent trials required for $n$ successes) and (7.4) is the same geometric distribution (the number of independent trials required for first success). Therefore, we call (7.3) the delta negative binomial distribution and its special case (7.2) is the delta geometric distribution. Then, the delta discrete exponential distribution is the same delta geometric distribution. 
(c) For $\alpha=\frac{n}{2}, n \in \mathbb{N}$, and $\beta=\frac{1}{2}, \Gamma^{\Delta}(\alpha, \beta)$ in (7.1) is delta discrete Chi-square distribution, $\chi^{2 \Delta}$ with pmf

$$
\operatorname{Pr}[X=x]=\frac{x^{\frac{n}{2}-1}}{\Gamma\left(\frac{n}{2}\right) 2^{\frac{n}{2}}}\left(\frac{2}{3}\right)^{\sigma(x)}, \quad x=\frac{n}{2}-1, \frac{n}{2}, \ldots .
$$

In the special case $n=2$, we obtain delta discrete exponential distribution, i.e.

$$
\operatorname{Pr}[X=x]=\left(\frac{1}{2}\right)\left(\frac{3}{2}\right)^{-\sigma(x)}, \quad x=0,1, \ldots
$$

- Statistical properties:

Theorem 7.2. If $X \sim \Gamma^{\Delta}(\alpha, \beta)$, then the expectation, variance and delta moment generating function of the random variable $X$ are given by

$$
\begin{aligned}
& E[X]=\alpha(1+\beta) \beta^{-1}-1, \\
& \operatorname{Var}(X)=\alpha(1+\beta) \beta^{-2}, \\
& M_{\sigma(X)}(t)=\left(\frac{1}{1-t(1+\beta) \beta^{-1}}\right)^{\alpha} .
\end{aligned}
$$

Proof. We have

$$
E[X]=\sum_{x=\alpha-1}^{\infty} \frac{x x^{\frac{\alpha-1}{\beta}} \beta^{\alpha}}{\Gamma(\alpha)(1+\beta)^{\sigma(x)}}
$$

by use of the relation $(x-\alpha+1) x^{\underline{\alpha-1}}=x^{\underline{\alpha}}$, we have

$$
E[X]=\frac{\beta^{\alpha}}{\Gamma(\alpha)} \sum_{x=\alpha-1}^{\infty} \frac{x^{\underline{\alpha}}}{(1+\beta)^{\sigma(x)}}+(\alpha-1) .
$$

On the other hand, we have

$$
\begin{aligned}
\sum_{x=\alpha-1}^{\infty} \frac{x^{\underline{\alpha}}}{(1+\beta)^{\sigma(x)}} & =\sum_{x=\alpha}^{\infty}\left(\frac{1}{1+\beta}\right)^{\sigma(x)} \frac{\Gamma(x+1)}{\Gamma(x+1-\alpha)} \\
& =\left(\frac{1}{1+\beta}\right)^{1+\alpha} \sum_{x=0}^{\infty}\left(\frac{1}{1+\beta}\right)^{x} \frac{\Gamma(x+\alpha+1)}{\Gamma(x+1)}
\end{aligned}
$$

Now, we apply theorem 2.2.1 from [4] to the right-hand side and introduce the hypergeometric function ${ }_{2} F_{1}$ in the following way

$$
\sum_{x=\alpha-1}^{\infty} \frac{x^{\underline{\alpha}}}{(1+\beta)^{\sigma(x)}}=\left(\frac{1}{1+\beta}\right)^{1+\alpha} \Gamma(1+\alpha)_{2} F_{1}\left(1,1+\alpha ; 1 ; \frac{1}{1+\beta}\right),
$$


by using exercise 4.2.10 from [7] to the right-hand side, we have

$$
\begin{aligned}
\sum_{x=\alpha-1}^{\infty} \frac{x^{\underline{\alpha}}}{(1+\beta)^{\sigma(x)}} & =\left(\frac{1}{1+\beta}\right)^{1+\alpha} \frac{1}{\Gamma(-\alpha)} \int_{0}^{1} x^{\alpha}(1-x)^{-\alpha-1}\left(1-\frac{x}{1+\beta}\right)^{-1} d x \\
& =\left(\frac{1}{1+\beta}\right)^{\alpha} \frac{1}{\Gamma(-\alpha)} \int_{0}^{1} \frac{(1-x)^{\alpha} x^{-\alpha-1}}{x+\beta} d x \\
& =\left(\frac{1}{1+\beta}\right)^{\alpha} \frac{1}{\Gamma(-\alpha)} B(1+\alpha,-\alpha)(1+\beta)^{\alpha} \beta^{-(\alpha+1)} \\
& =\frac{\Gamma(\alpha+1)}{\beta^{\alpha+1}},
\end{aligned}
$$

where $B(.,$.$) is the ordinary beta function. Then we have$

$$
\sum_{x=\alpha-1}^{\infty} \frac{x^{\underline{\alpha}}}{(1+\beta)^{\sigma(x)}}=\frac{\Gamma(\alpha+1)}{\beta^{\alpha+1}}
$$

and which complete the proof of (7.8). For the proof of (7.9) we have

$$
\begin{aligned}
E\left[X^{2}\right] & =\sum_{x=\alpha-1}^{\infty} \frac{x^{2} x^{\underline{\alpha-1}} \beta^{\alpha}}{\Gamma(\alpha)(1+\beta)^{\sigma(x)}} \\
& =\frac{\beta^{\alpha}}{\Gamma(\alpha)} \sum_{x=\alpha-1}^{\infty}\left(\frac{1}{1+\beta}\right)^{\sigma(x)} x\left(x^{\underline{\alpha}}+(\alpha-1) x^{\underline{\alpha-1}}\right) \\
& =\frac{\beta^{\alpha}}{\Gamma(\alpha)} \sum_{x=\alpha-1}^{\infty}\left(\frac{1}{1+\beta}\right)^{\sigma(x)} x x^{\underline{\alpha}}+(\alpha-1)\left(\alpha\left(1+\beta^{-1}\right)-1\right)
\end{aligned}
$$

since $(1+x) x^{\underline{\alpha}}=(1+x)^{\underline{\alpha+1}}$, which implies that

$$
\sum_{x=\alpha-1}^{\infty} \frac{x x^{\underline{\alpha}}}{(1+\beta)^{\sigma(x)}}=\sum_{x=\alpha-1}^{\infty} \frac{(1+x)^{\underline{1+\alpha}}}{(1+\beta)^{\sigma(x)}}-\sum_{x=\alpha-1}^{\infty} \frac{x^{\underline{\alpha}}}{(1+\beta)^{\sigma(x)}}
$$

and by considering (7.11), we get

$$
\sum_{x=\alpha-1}^{\infty} \frac{(1+x)^{\frac{1+\alpha}{\alpha}}}{(1+\beta)^{\sigma(x)}}=\frac{(1+\beta) \Gamma(\alpha+2)}{\beta^{\alpha+2}}
$$

and from which, we have

$$
E\left[X^{2}\right]=2 \alpha(\alpha-1) \beta^{-1}+\alpha(\alpha+1) \beta^{-2}+(\alpha-1)^{2} .
$$


Also, with

$$
\begin{aligned}
M_{\sigma(X)}(t)= & \sum_{x=\alpha-1}^{\infty}\left(\frac{1}{1-t}\right)^{\sigma(x)} \frac{x^{\frac{\alpha-1}{}} \beta^{\alpha}}{\Gamma(\alpha)(1+\beta)^{\sigma(x)}} \\
& =\sum_{x=\alpha-1}^{\infty} \frac{x^{\frac{\alpha-1}{2}} \beta^{\alpha}}{\Gamma(\alpha)((1-t)(1+\beta))^{\sigma(x)}} \\
& =\sum_{x=\alpha-1}^{\infty} \frac{x^{\frac{\alpha-1}{}} \beta^{\alpha}}{\Gamma(\alpha)\left((1+(\beta-t(1+\beta)))^{\sigma(x)}\right.} \\
& =\left(\frac{1}{1-t\left(1+\beta^{-1}\right)}\right)^{\alpha},
\end{aligned}
$$

the proof is complete.

Also, it is easily seen from (7.11) that

$$
\sum_{x=\alpha-1}^{\infty} \frac{x^{\frac{\alpha-1}{\beta}} \beta^{\alpha}}{\Gamma(\alpha)(1+\beta)^{\sigma(x)}}=1, \quad \alpha>0, \beta>0 .
$$

- Maximum Likelihood Estimation (MLE):

Let $x_{1}, x_{2}, \ldots, x_{n}$ be a random sample.If this sample are assumed to be independently and identically distributed (iid) random variables following $\Gamma^{\Delta}(\alpha, \beta)$ distribution, then the likelihood function of the sample will be

$$
L=\frac{\beta^{n \alpha} \prod x_{i}^{\underline{\alpha-1}}}{\Gamma^{n}(\alpha)(1+\beta)^{n+\Sigma x_{i}}} I_{\{\alpha-1, \alpha, \ldots\}}\left(x_{i}\right)
$$

\subsection{The nabla discrete gamma distribution}

Definition 7.3. It is said that the random variable $X$ has a nabla discrete gamma distribution with $(\alpha, \beta)$ parameters if its pmf is given by

$$
\operatorname{Pr}[X=x]=\frac{h_{\overline{\alpha-1}}(x) \beta^{\alpha}}{e_{\beta}^{*}(\rho(x), 0)}=\frac{x^{\overline{\alpha-1}} \beta^{\alpha}(1-\beta)^{\rho(x)}}{\Gamma(\alpha)}, \quad x=\mathbb{N}_{1},
$$

where $\alpha>0,0<\beta<1$ and it denotes as $\Gamma^{\nabla}(\alpha, \beta)$.

- Particular cases:

(a) For $\alpha=1, \Gamma^{\nabla}(\alpha, \beta)$ in (7.13) reduces to a one parameter nabla discrete gamma or nabla exponential distribution, $\Gamma^{\nabla}(1, \beta) \equiv E^{\nabla}(\beta)$ with pmf

$$
\operatorname{Pr}[X=x]=\beta(1-\beta)^{\rho(x)}, \quad x=1,2, \ldots .
$$

Obviously, this is the pmf of geometric distribution (the number of independent trials required for first success). 
(b) For $\alpha=n, n \in \mathbb{N}, \Gamma^{\nabla}(\alpha, \beta)$ in (7.13) is an nabla discrete Erlang distribution $\Gamma^{\nabla}(n, \beta)$ with pmf

$$
\operatorname{Pr}[X=x]=\left(\begin{array}{c}
x+n-2 \\
x-1
\end{array}\right) \beta^{n}(1-\beta)^{\rho(x)}, \quad x=\mathbb{N}_{1}
$$

If we substitute $\rho(x)=x,(7.14)$ and (7.15) are given by

$$
\operatorname{Pr}[X=x]=\beta(1-\beta)^{x}, \quad x=0,1, \ldots, .
$$

and

$$
\operatorname{Pr}[X=x]=\left(\begin{array}{c}
x+n-1 \\
x
\end{array}\right) \beta^{n}(1-\beta)^{x}, \quad x=0,1, \ldots,
$$

respectively. It can be seen that (7.17) is the same negative binomial distribution (the number of failures for $n$ successes) and (7.16) is the same geometric distribution (the number of failures for first success). Then, we call (7.15) the nabla negative binomial distribution and its special case (7.14) is the nabla geometric distribution. Therefore the nabla discrete exponential distribution is the same nabla geometric distribution.

(c) For $\alpha=\frac{n}{2}, n \in \mathbb{N}$ and $\beta=\frac{1}{2}, \quad \Gamma^{\nabla}(\alpha, \beta)$ in (7.13) is nabla discrete Chi-square distribution, $\chi^{2 \nabla}$ with pmf

$$
\operatorname{Pr}[X=x]=\frac{x^{\frac{n}{2}-1}}{\Gamma\left(\frac{n}{2}\right) 2^{\frac{n}{2}+\rho(x)}}, \quad x=1,2, \ldots .
$$

In the special case $n=2$, we obtain nabla discrete exponential distribution, i.e.

$$
\operatorname{Pr}[X=x]=\left(\frac{1}{2}\right)^{x}, \quad x=1,2, \ldots
$$

- Statistical properties:

Theorem 7.4. If $X \sim \Gamma^{\nabla}(\alpha, \beta)$, then the expectation, variance and nabla moment generating function of the random variable of $X$ are given by

$$
\begin{aligned}
& E[X]=\alpha(1-\beta) \beta^{-1}+1, \\
& \operatorname{Var}(X)=\alpha(1-\beta) \beta^{-2}, \\
& M_{\rho(X)}(t)=\left(\frac{1}{1-t(1-\beta) \beta^{-1}}\right)^{\alpha} .
\end{aligned}
$$

Proof. We have

$$
E[X]=\sum_{x=1}^{\infty} \frac{x x^{\overline{\alpha-1}} \beta^{\alpha}(1-\beta)^{\rho(x)}}{\Gamma(\alpha)}
$$


since $(x+\alpha-1) x^{\overline{\alpha-1}}=x^{\bar{\alpha}}$, it results

$$
E[X]=\frac{\beta^{\alpha}}{\Gamma(\alpha)} \sum_{x=1}^{\infty} x^{\bar{\alpha}}(1-\beta)^{\rho(x)}+(1-\alpha)
$$

On the other hand, with similar method for the proof of theorem 7.2, we have

$$
\begin{aligned}
\sum_{x=1}^{\infty} x^{\bar{\alpha}}(1-\beta)^{\rho(x)} & =\sum_{x=0}^{\infty}(1-\beta)^{x} \frac{\Gamma(x+\alpha+1)}{\Gamma(x+1)} \\
& =\Gamma(\alpha+1)_{2} F_{1}(1,1+\alpha ; 1 ; 1-\beta) \\
& =\frac{1}{\Gamma(-\alpha)} \int_{0}^{1} \frac{x^{-\alpha-1}(1-x)^{\alpha}}{x+\beta(1-x)} d x \\
& =\frac{\Gamma(1+\alpha)}{\beta^{\alpha+1}}
\end{aligned}
$$

Here, we applied the following identity from [4],

$$
\int_{0}^{1} \frac{x^{\alpha-1}(1-x)^{\beta-1}}{(a x+b(1-x))^{\alpha+\beta}} d x=\frac{\Gamma(\alpha) \Gamma(\beta)}{a^{\alpha} b^{\beta} \Gamma(\alpha+\beta)} .
$$

Then we obtain

$$
\sum_{x=1}^{\infty} x^{\bar{\alpha}}(1-\beta)^{\rho(x)}=\frac{\Gamma(\alpha+1)}{\beta^{\alpha+1}}
$$

and this complete the proof of (7.20). For the proof of (7.21), we have

$$
\begin{aligned}
E\left[X^{2}\right] & =\sum_{x=1}^{\infty} \frac{x^{2} x^{\overline{\alpha-1}}(1-\beta)^{\rho(x)} \beta^{\alpha}}{\Gamma(\alpha)} \\
& =\frac{\beta^{\alpha}}{\Gamma(\alpha)} \sum_{x=1}^{\infty}(1-\beta)^{\rho(x)} x\left(x^{\bar{\alpha}}+(1-\alpha) x^{\overline{\alpha-1}}\right) \\
& =\frac{\beta^{\alpha}}{\Gamma(\alpha)} \sum_{x=1}^{\infty}(1-\beta)^{\rho(x)} x x^{\bar{\alpha}}+(1-\alpha)\left(\alpha(1-\beta) \beta^{-1}+1\right),
\end{aligned}
$$

since $x^{\overline{\alpha+1}}=(x+\alpha) x^{\bar{\alpha}}$, we have

$$
\sum_{x=1}^{\infty} x x^{\bar{\alpha}}(1-\beta)^{\rho(x)}=\sum_{x=1}^{\infty} x^{\overline{\alpha+1}}(1-\beta)^{\rho(x)}-\alpha \sum_{x=1}^{\infty} x^{\bar{\alpha}}(1-\beta)^{\rho(x)}
$$

now by considering (7.23),

$$
E\left[X^{2}\right]=\alpha(1+\alpha) \beta^{-2}-\alpha^{2} \beta^{-1}+(1-\alpha)\left(\alpha(1-\beta) \beta^{-1}+1\right) .
$$


Also, with

$$
\begin{aligned}
M_{\rho(X)}(t)= & \sum_{x=1}^{\infty}(1+t)^{\rho(x)} \frac{x^{\overline{\alpha-1}} \beta^{\alpha}(1-\beta)^{\rho(x)}}{\Gamma(\alpha)} \\
& =\sum_{x=1}^{\infty} \frac{x^{\overline{\alpha-1}} \beta^{\alpha}((1+t)(1-\beta))^{\rho(x)}}{\Gamma(\alpha)} \\
& =\sum_{x=1}^{\infty} \frac{x^{\overline{\alpha-1}} \beta^{\alpha}\left((1-(\beta-t(1-\beta)))^{\rho(x)}\right.}{\Gamma(\alpha)} \\
& =\left(\frac{1}{1-t\left(\beta^{-1}-1\right)}\right)^{\alpha},
\end{aligned}
$$

the proof is complete.

Also, it is easily seen from (7.23) that

$$
\sum_{x=1}^{\infty} \frac{x^{\overline{\alpha-1}} \beta^{\alpha}(1-\beta)^{\rho(x)}}{\Gamma(\alpha)}=1, \quad \alpha>0,0<\beta<1 .
$$

- Maximum Likelihood Estimation (MLE):

Let $x_{1}, x_{2}, \ldots, x_{n}$ be a random sample.If this sample are assumed to be independently and identically distributed (iid) random variables following $\Gamma^{\nabla}(\alpha, \beta)$ distribution, then the log likelihood function of the sample will be

$$
\log L=n \alpha \log \beta+\left(\sum x_{i}-n\right) \log (1-\beta)-n \log \Gamma(\alpha)+\sum \log x_{i}^{\overline{\alpha-1}} .
$$

\section{Unification of the continuous and discrete gamma distributions}

For a given time scale $\mathbb{T}$, we present the construction of pdf of gamma distribution, such that, the density function on time scales is

$$
f_{X}(x)=\frac{\hat{h}_{\alpha-1}(x) \beta^{\alpha}}{\hat{e}_{\beta}(\eta(x), 0)}, \quad x \in \mathbb{T} .
$$

In order that, the reader sees how the pdf of continuous gamma distribution and delta and nabla discrete gamma distributions follow from (8.1), it is only at this point necessary to know that

$$
\begin{aligned}
& \hat{h}_{\alpha-1}(x)=h_{\alpha-1}(x)=\frac{x^{\alpha-1}}{\Gamma(\alpha)}, \quad \eta(x)=x \quad \text { and } \quad \hat{e}_{\beta}(\eta(X), 0)=e^{\beta x} \quad \text { if } \mathbb{T}=\mathbb{R}^{+}, \\
& \hat{h}_{\alpha-1}(x)=h_{\underline{\alpha-1}}(x)=\frac{x^{\underline{\alpha-1}}}{\Gamma(\alpha)}, \quad \eta(x)=\sigma(x) \quad \text { and } \quad \hat{e}_{\beta}(\eta(X), 0)=(1+\beta)^{\sigma(x)}
\end{aligned}
$$

if $\mathbb{T}=\mathbb{N}_{\alpha-1}, \alpha>0$. If $\mathbb{T}=\mathbb{N}_{1}$ then we have

$$
\hat{h}_{\alpha-1}(x)=h_{\overline{\alpha-1}}(x)=\frac{x^{\overline{\alpha-1}}}{\Gamma(\alpha)}, \quad \eta(x)=\rho(x) \quad \text { and } \quad \hat{e}_{\beta}(\eta(X), 0)=(1-\beta)^{-\rho(x)} .
$$




\section{Application}

Here, the data give the time to the death (in week) of AG positive leukemia patients ( see [18] and [19] ).

$\{65,156,100,134,16,108,121,4,39,143,56,26,22,1,1,5,65\}$

Table 1: Results

\begin{tabular}{ccccc}
\hline \hline Model & MLE $(\mathrm{s})$ & $\hat{\log L}$ & $\mathrm{AIC}$ & $\mathrm{BIC}$ \\
\hline$\Gamma^{\Delta}(\alpha, \beta)$ & $\hat{\alpha}=2, \hat{\beta}=0.0330739$ & -93.8669 & 191.734 & 193.4 \\
$\operatorname{Zipf}(\theta, n=500)$ & $\hat{\theta}=0.1484037$ & -101.148 & 206.296 & 207.962 \\
$\operatorname{Zeta}(\gamma)$ & $\hat{\gamma}=1.439751$ & -100.276 & 202.552 & 203.385
\end{tabular}

The pmfs of Zeta and Zipf considered here for fitting are, respectively, given by

$$
P(X=x)=\frac{1}{x^{\gamma} \sum_{i=1}^{\infty}\left(\frac{1}{i}\right)^{\gamma}}, \quad x=1,2, \ldots
$$

and

$$
P(X=x)=\frac{1}{x^{\theta} \sum_{i=1}^{n}\left(\frac{1}{i}\right)^{\theta}}, \quad x=1,2, \ldots, n .
$$

Let $x_{1}, x_{2}, \ldots, x_{m}$ be a random sample.If this sample are assumed to be independently and identically distributed (iid) random variables following Zeta or Zipf distributions, then the log likelihood function of the sample will be

$$
\log L=-\gamma \sum_{i=1}^{m} \log x_{i}-m \log \sum_{i=1}^{\infty}\left(\frac{1}{i}\right)^{\gamma}
$$

or $\log \mathrm{L}=-\theta \sum_{i=1}^{m} \log x_{i}-m \log \sum_{i=1}^{n}\left(\frac{1}{i}\right)^{\theta}$, respectively.

\section{Acknowledgement}

We would like to thank the referees for a careful reading of our paper and lot of valuable suggestions on the first draft of the manuscript. 


\section{Appendix}

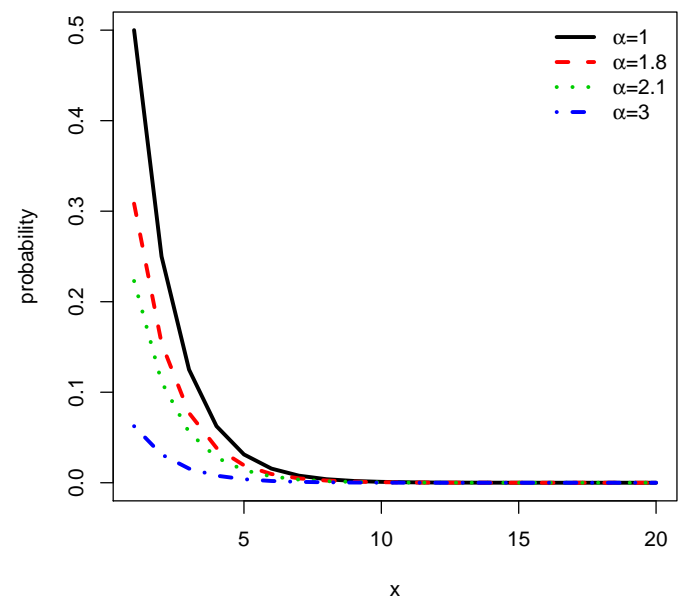

Figure 1: Probability mass functions $\left(\Gamma^{\Delta}\right)$ for various values of $\alpha$ and $\beta=0.5$

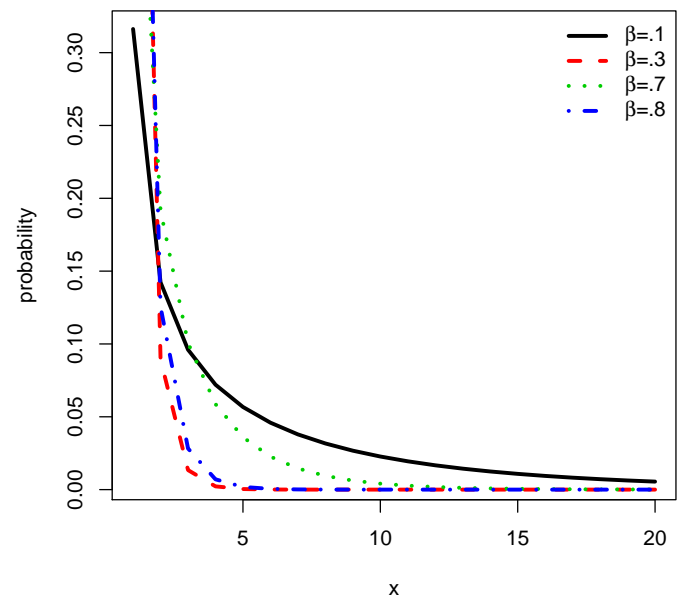

Figure 2: Probability mass functions $\left(\Gamma^{\Delta}\right)$ for various values of $\beta$ and $\alpha=0.5$ 


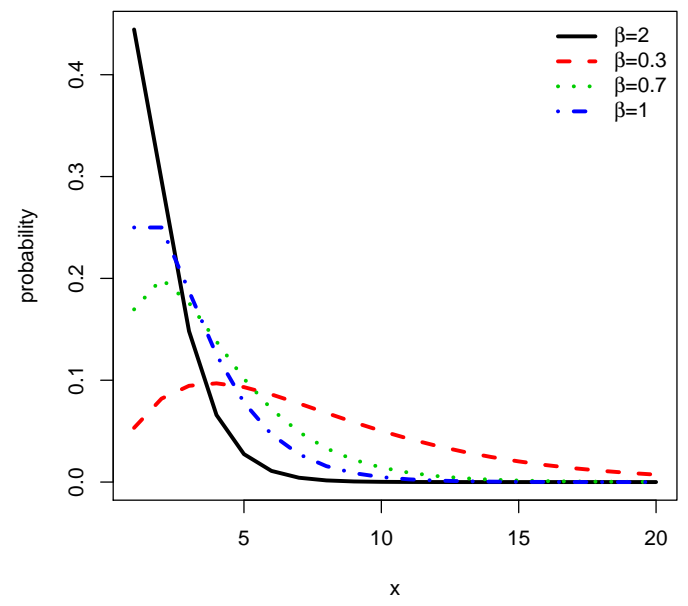

Figure 3: Probability mass functions $\left(\Gamma^{\Delta}\right)$ for various values of $\beta$ and $\alpha=2$

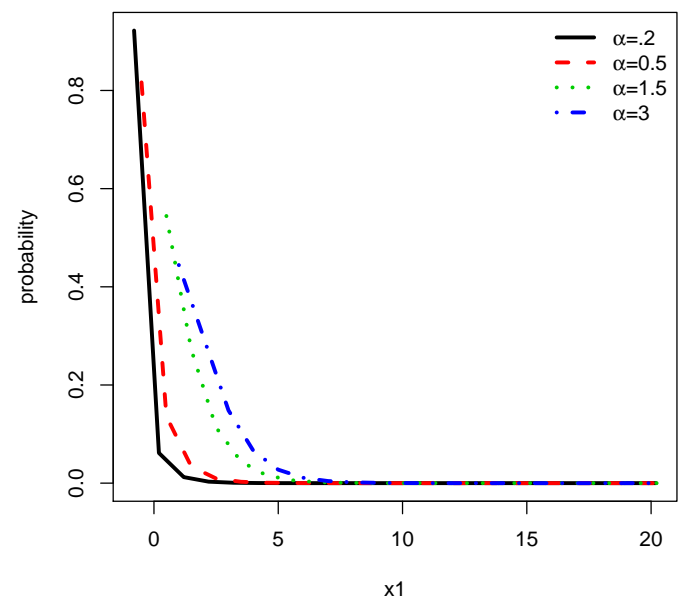

Figure 4: Probability mass functions $\left(\Gamma^{\Delta}\right)$ for various values of $\alpha$ and $\beta=2$

\section{References}

[1] T. Abdeljawad, Dual identities in fractional difference calculus within Riemann, Advances in Differences Equations, Vol. 2013, article 36, 2013.

[2] T. Abdeljawad, fractional sums and differences with Binomial cofficients, Discrete Dynamics in Nature and Society, Vol. 2013, Article ID 104173, 6 pages, 2013.

[3] A. M. Abouammoh and N. S. Alhazzani, On discrete gamma distribution, Commun. Statist. Theor. Meth. 44, 3087-3098, 2015. 
[4] G. E. Andrews, R. Askey and R. Roy, Special functions, volume 71 of Encyclopedia of Mathematics and its Applications, Cambridge University Press, Cambridge, 1999.

[5] M. Bohner and A. Peterson, Dynamic Equations on Time Scales, Birkhuser, Boston, 2001.

[6] M. Bohner and A. Peterson, Advances in Dynamic Equations on Time Scales, Birkhuser, Boston, 2003.

[7] B. C. Carlson, Special functions of applied mathematics, Academic Press [Harcourt Brace Jovanovich Publishers], New York, 1977.

[8] S. Chakraborty and D. Chakravarty, Discrete gamma distributions: properties and parameter estimations, Commun. Statist. Theor. Meth. 41, 3301-3324, 2012.

[9] S. Chakraborty, Generating discrete analogues of continuous probability distributions-A survey of methods and constructions, Journal of Statistical Distributions and Applications (2015) 2:6 DOI 10.1186/s40488-015-0028-6, 1-30.

[10] M. Ganji and F. Gharari, The Generalized Random Variable Appears the Trace of Fractional Calculus in Statistics, Applied Mathematics Information Sciences Letters, 3 (2), 61-67, 2015.

[11] M. Holm, The theory of discrete fractional calculus development and application [dissertation], University of Nebraska, Lincoln, Neb, USA, 2011.

[12] M. A. Jazi, C. D. Lai and M. H. Alamatsaz, A discrete inverse Weibull distribution and estimation of its parameters, Statist. Methodol. 7, 121-132, 2009.

[13] A. W. Kemp, The discrete half-normal distribution, Int. Conf. Mathemat. Statist. Model. Honor of Enrique Castillo, June 28-30, 2008.

[14] M. S. Khan, A. Khalique and A. M. Aboummoh, On estimating parameters in a discrete Weibull distribution, IEEE Trans. Reliab. 38(3), 348-350, 1989.

[15] H. Krishna and P. S. Pundir, Discrete Burr and discrete Pareto distributions, Methodol. 6, 177-188, 2009.

[16] T. Nakagawa and S. Osaki, The discrete Weibull distribution, IEEE Transact. Reliab. R-24(5), 300-301, 1975.

[17] D. Roy The discrete normal distribution, Commun. Statist. Theor. Meth. 32(10), 1871-1883, 2003.

[18] P. Feigl, M. Zelen, Estimation of exponential survival probabilities with concomitant information, Biometrics 21, 826-838, 1965.

[19] D. J. Hand, F. Daly, A. D. Lunn, K. J. McConway and E. O. Ostrowski, A Hand Book of Small Data Sets, London: Chapman and Hall, 1994. 